Classification

Physics Abstracts

$61.30-05.50$

\title{
Hard rods on lattices
}

\author{
A. Baumgärtner \\ Institut für Festkörperforschung der Kernforschungsanlage Jülich, D-5170 Jülich, F.R.G
}

(Reçu le 11 mars 1985, accepté sous forme définitive le 10 juin 1985)

\begin{abstract}
Résumé. - Ce travail présente des calculs exacts et des résultats d'énumération pour des barres rigides distribuées à densité maximale sur des réseaux carrés $L \times L$. Chaque barre occupe $N<L$ sites voisins. Contrairement aux théories de champ moyen, nos calculs - bien que restreints à des valeurs finies de $L$ et de $N$ - permettent d'éliminer l'existence d'un ordre orientationnel à longue distance : en effet la valeur absolue du paramètre d'ordre orientationnel est $\langle|s|\rangle \leqslant 11 / 36$ pour $L / N>2$ et décroît continûment pour tout $N$ lorsque le rapport $L / N$ augmente. L'entropie par barre de nos systèmes se comporte comme $S_{N} \propto N^{-1} \ln N$ lorsque $N \rightarrow \infty$. Les barres rigides sur des réseaux cubiques sont aussi brièvement discutées. Nous discutons quelques implications de nos résultats sur la physique des cristaux liquides, des cristaux liquides polymériques et sur les théories actuelles de la fusion des polymères.
\end{abstract}

\begin{abstract}
Exact calculations and enumerations of systems of hard rigid rods distributed at closed packed density on $L \times L$ square lattices, where each rod occupies $N<L$ nearest neighbour lattice sites, have been performed. In contrast to mean-field theories our results though restricted to finite $L$ and $N$ provide evidence against long-range orientational order : the absolute magnitude of the orientational order parameter is $\langle|s|\rangle \leqslant 11 / 36$ for $L / N\rangle 2$ and decreases continuously with increasing size of the system $L / N$ for all $N$. The entropy per rod of our systems behaves as $S_{N} \propto N^{-1} \times$ $\ln N$ as $N \rightarrow \infty$. Hard rods on cubic lattices are also briefly considered. Some implications of our results are discussed with respect to liquid crystals, polymeric liquid crystals and current theories of polymer melting.
\end{abstract}

\section{Introduction.}

Strict lattice models have been used in theories of orientational ordering phenomena as for example for liquid crystals of inflexible rods [1-3], for polymer liquid crystals and polymer crystallization [3-7]. In these models the number of allowed orientations of rodlike molecules is finite. Each rod occupies a row of $N$ consecutive lattice sites. The rod can point only in the directions defined by the nearest-neighbour lattice vectors. Despite the unrealistic discrete rotational symmetric properties, as compared to real rodlike molecules with continuous distribution of orientations, the strict lattice model has served as a useful model to understand the equilibrium packing statistics of macromolecules.

It is not yet understood whether lattice models have similar equilibrium statistical properties as continuous models, or even belong to the same universality class in case of critical phenomena as compared with continuous models.

However, continuous models for rodlike molecules [8,9] investigated using mean-field methods reveal discontinuous transitions between the isotropic and the nematic state depending on density 
and length of the rod, which is in qualitative agreement with experimental facts; and in fact corresponding phase transitions are reported for strict lattice models $[1-4,7]$ too.

But on the other hand, it has not yet been proved that lattice models actually lead to a transition. Dimers $(N=2)$ on the square lattice are known to show no ordering even at closed packed density [10-12].

In fact recent Monte Carlo calculations of semiflexible polymers on square and cubic lattices [13] indicated that their ground state configurations consisting of hard rods are highly degenerated exhibiting ordered domains at equilibrium, which is at variance with what is known of molecular order in nematic liquid crystals.

In the present work we have examined rigorously the statistics of hard rods on the square lattice at closed packed density. The rods are distributed on $L \times L$ lattices with edges. Each rod occupies $N<L$ consecutive nearest-neighbour lattice sites. The aim of the present work is to calculate exact values of orientational order parameter and entropy for small systems in order to obtain from systematic dependencies on $L / N$ and $N$ some informations clarifying to some extent the question of existence or non-existence of an ordered phase.

\section{Results.}

Our first result is that for $L / N=2$ the total number of configurations is given by

$$
Z_{N}=2(N-1)^{2}+2(N+3)^{2}-2^{4}=4(N+1)^{2} .
$$

The term $(N+3)^{2}$ in (1) gives the number of such configurations which can be splitted up (without " breaking " rods) into two strips of size $N \times L$. Each strip exhibit $N+3$ configurations. The degeneracy factor 2 in front of this term needs a correction, the last term $2^{4}$, due to double counting of configurations which can be splitted up in four cells of size $N \times N$. The first term $(N-1)^{2}$ gives the number of such " mixed " configurations which cannot be reduced to cells of linear sizes compatible with $N$. This term have been found from a systematic analysis of configurations with increasing $N$. These « mixed " configurations are the main obstacle preventing a derivation of a formula of $Z_{N}$ for $L / N>2$.

The orientational order parameter $s$, which is defined as the difference between the fraction of rods pointing in horizontal and in vertical directions is given by

$$
\begin{aligned}
& \langle|s|\rangle=\left[(N-1)^{2}+4 N+2\right] / Z_{N} \\
& \left\langle s^{2}\right\rangle=\left[(N-1)^{2} / 2+2(N+3)-4\right] / Z_{N}
\end{aligned}
$$

The various terms in (2) and (3) have been calculated according to the procedure given above for equation (1). The entropy per rod is according to (1) for $N \rightarrow \infty$

$$
S_{N}=(4 n)^{-1} \ln Z_{N} \simeq(2 N)^{-1} \ln N
$$

Some numbers according to equations (1) to (4) are given in tables I to IV and are plotted in figures 1 and 2.

Other data presented there have been calculated by exact enumerations using the computer. It should be noted that exact enumeration results coincide with equations (1)-(4) in the case of $L / N=2$, and with exact data given in reference [1,0] for $N=2$, which proves the reliability of our numerical procedure. The results for $N=4,5$ with $L / N=4$ are not exact, but are estimated using a special extrapolation procedure. Exact calculations would require more than 200 hours on the Cray X-MP.

The extrapolation method is as follows. In the first step, the lattice is filled up successively with rods from the bottom line up to a row $r<L$. In the original procedure for exact enumerations we 


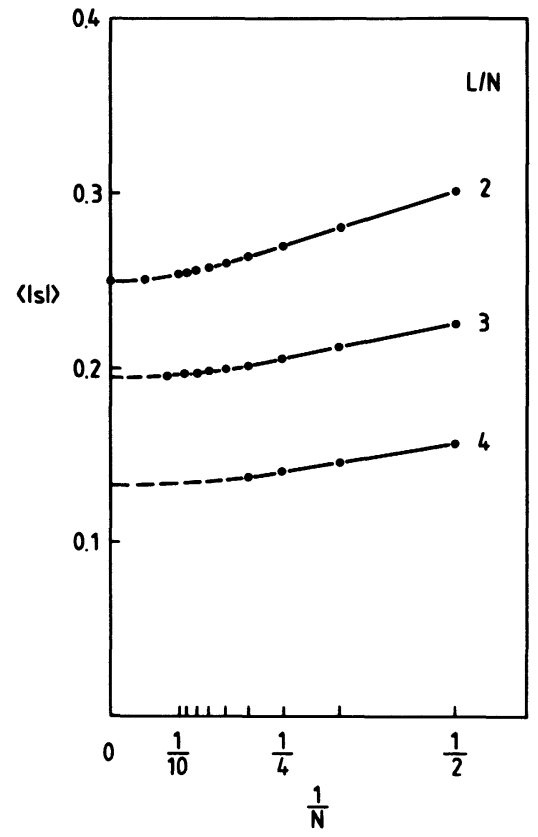

Fig. 1. - Absolute magnitude of the orientational order parameter $\langle|s|\rangle$ versus $N^{-1}$ for $L / N=2,3,4$.

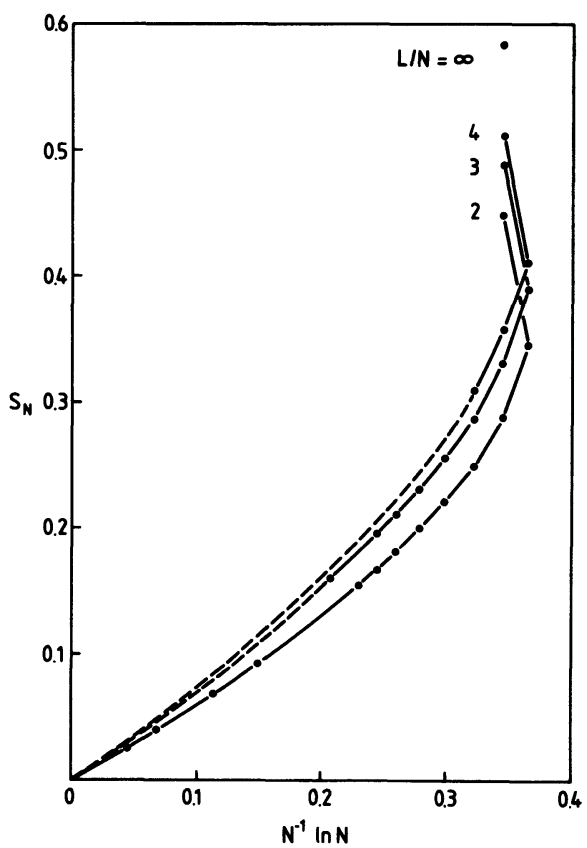

Fig. 2. - Entropy per rod $S_{N}$ versus $N^{-1} \ln N$ for $L / N=2,3,4$. The value of $S_{N}$ for $L / N=\infty$ is taken from Refs. [10,11]. 
Table I. - Total number of configurations $Z_{N}$ for rods of lengths $N$ on several $L \times L$ square lattices.

\begin{tabular}{rrrrr}
\multicolumn{1}{r}{$L / N$} & 2 & \multicolumn{1}{c}{3} & \multicolumn{1}{c}{4} & $\infty$ \\
\hline$N=2$ & 36 & 6728 & 12988816 & $\infty$ \\
3 & 64 & 37160 & 378875648 & \\
4 & 100 & 135950 & $\left(8.37 \times 10^{9}\right)$ & \\
5 & 144 & 389216 & $\left(5.89 \times 10^{10}\right)$ & \\
6 & 196 & 945352 & & \\
7 & 256 & 2039288 & & \\
8 & 324 & 4020710 & & \\
9 & 400 & 7386240 & & \\
10 & 484 & - & & \\
11 & 576 & - & & \\
12 & 676 & 33744398 & & \\
$:$ & $\vdots$ & & & \\
$\infty$ & $\infty$ & & & \\
\hline
\end{tabular}

Table II. - Entropy per rod $S_{N}=\ln Z_{N} /\left(L^{2} / N\right)$.

\begin{tabular}{rcccc}
$L / N$ & 2 & 3 & 4 & $\infty$ \\
\hline$N=2$ & 0.448 & 0.4897 & 0.512 & 0.5831 \\
3 & 0.346 & 0.3897 & 0.4115 & \\
4 & 0.288 & 0.3283 & $(0.357 \pm 0.01)$ & \\
5 & 0.248 & 0.2860 & $(0.310 \pm 0.02)$ & \\
6 & 0.220 & 0.2548 & & \\
7 & 0.198 & 0.2306 & & \\
8 & 0.181 & 0.2112 & & \\
9 & 0.166 & 0.1952 & & \\
10 & 0.154 & - & & \\
11 & 0.144 & - & & \\
12 & 0.136 & 0.1605 & & \\
$\vdots$ & $:$ & & & \\
$\infty$ & 0 & & &
\end{tabular}

would continue up to $r=L$; but now already at row $r$ the number of " preliminary " configurations is so large that we could not store any more such configurations because adding a new rod to these uncompleted configurations would increase their number $z$ again by about a factor of two. Therefore, as a next step in our procedure, only some of these configurations (selected at random) have been completed by exact enumerations. By plotting our quantities of interest, $\langle|s|\rangle$ and $S_{N}$, versus the fraction $x / z$ of completed configurations one can obtain from extrapolation to $x / z=1$ a fairly good estimate of $\langle|s|\rangle$ and $S_{N}$ (Fig. 3). The accuracy of this method has been compared with exact known results. 
Table III. - Absolute magnitude of the orientational order-parameter $\langle|s|\rangle$

\begin{tabular}{rcccc}
$L / N$ & 2 & 3 & 4 & $\infty$ \\
\hline$N=2$ & 0.305 & 0.2247 & 0.1560 & 0 \\
3 & 0.281 & 0.2107 & 0.1452 & \\
4 & 0.270 & 0.2045 & $(0.140 \pm 0.02)$ & \\
5 & 0.264 & 0.2013 & $(0.139 \pm 0.03)$ & \\
6 & 0.260 & 0.1994 & & \\
7 & 0.258 & 0.1983 & & \\
8 & 0.256 & 0.1975 & & \\
9 & 0.255 & 0.1969 & & \\
10 & 0.254 & - & & \\
11 & 0.2535 & - & & \\
12 & 0.2529 & 0.1960 & & \\
$\vdots$ & $\vdots$ & & & \\
$\infty$ & $1 / 4$ & & & \\
\end{tabular}

Table IV. - Square root of the mean squared orientational order-parameter $\sqrt{\left\langle s^{2}\right\rangle}$.

\begin{tabular}{rcccc}
$L / N$ & 2 & 3 & 4 & $\infty$ \\
\hline$N=2$ & 0.425 & 0.2719 & 0.2012 & 0 \\
3 & 0.395 & 0.2532 & 0.1880 & \\
4 & 0.381 & 0.2450 & & \\
5 & 0.373 & 0.2407 & & \\
6 & 0.368 & 0.2382 & & \\
7 & 0.364 & 0.2366 & & \\
8 & 0.362 & 0.2356 & & \\
9 & 0.361 & 0.2348 & & \\
10 & 0.359 & - & & \\
11 & 0.3584 & - & & \\
12 & 0.3577 & 0.2337 & & \\
$\vdots$ & $\vdots$ & & \\
$\infty$ & $1 / \sqrt{8}$ & & \\
\hline
\end{tabular}

\section{Discussions and conclusions.}

The results presented in figures 1 and 2 exhibit that for lattice sizes $2 \leqslant L / N \leqslant 4$ (the trivial case $L=N$ excluded) the order parameter is $\langle|s|\rangle \leqslant 11 / 36$ and is continuously decreasing with increasing lattice sizes $L / N$. Since there is obviously no reason to expect that for larger $L / N$ the order parameter would change its behaviour drastically, one could expect $\langle|s|\rangle \rightarrow 0$ as $L / N \rightarrow \infty$ for any $N \geqslant 2$. But even without referring to this limiting behaviour, the present results though restricted to small systems are at variance with what is known of orientational order in, e.g., nematic liquid crystals. Hence, our main conclusion is that it seems very unlikely that for hard rods on square lattices there is any long-range orientational order. The present results are 


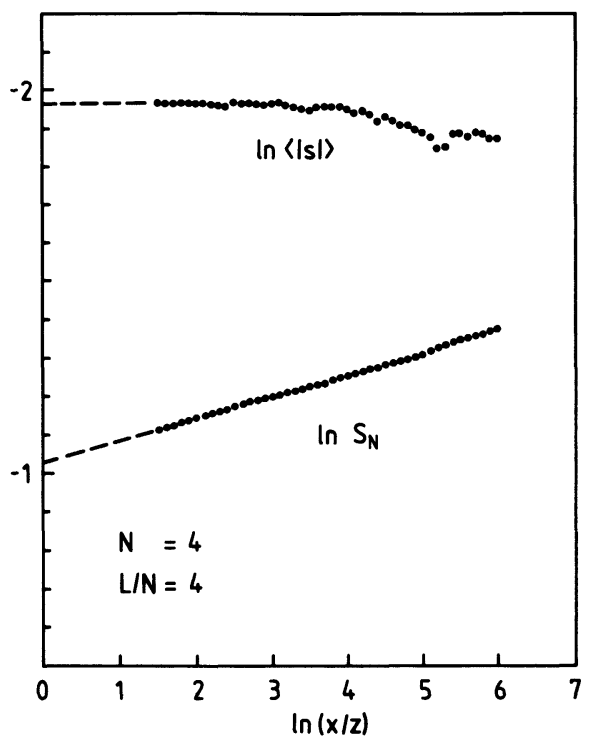

Fig. 3. - Log-log plot of the order parameter $\langle|s|\rangle$ and entropy $S_{N}$ versus fraction of completed configurations $x / z$.

also in agreement with previous findings [13] concerning the order parameter of larger systems $(L / N \leqslant 12)$ of almost-rigid rods $(N=10,20)$ on the square lattice with periodic boundary conditions which have been studied by Monte Carlo methods : those systems exhibit an order parameter less than 0.1 .

A second important argument against an ordered state is based on our results of the total number of configurations $Z_{N}$. According to table $\mathrm{I}$, or equivalently the entropy per rod presented in table II and figure 2, the number of configurations available for hard rods is huge, more similar to an isotropic state, and is increasing with increasing systems $L / N$, which again is at variance with what we expect of « crystalline " state. As a conjecture concluded from the present results (compare Fig. 2), we propose the limiting behaviour $S_{N}=N^{-1} \ln N$, or equivalently $Z_{N}=N^{(L / N)^{2}}$, for $L / N \rightarrow \infty$ and $N \rightarrow \infty$.

Of course, this conjecture is deduced from results on small systems with strict boundary conditions (" edges "); how far the conjecture would change by deducing it from small systems with periodic boundary conditions remains to be clarified, yet. However, using periodic boundary conditions would certainly increase considerably the number of configurations $Z_{N}$ at given $L / N$ and $N$, again supporting our main conclusion about the non-existence of an ordered phase.

The reason why we did not choose periodic boundary conditions in our calculations is precisely due to the enormous number of configurations even for small systems, which would have prevented the application of exact enumeration techniques. But since recently the question of orientational order in strict lattice models has become very controversial $[4,6]$, it is of some importance to have exact results at all, even restricted to small systems with strict boundary conditions.

The present results seem to raise some doubts on the reliability of mean-field lattice calculations predicting a transition between the isotropic and the anisotropic state [1-4, 7]. This criticism has to be understood not as a general one against lattice theories for macromolecules. The validity of lattice calculations applied to flexible polymers for instance has been demonstrated repeatedly by theories as well as by experiments. Their advantages and merits are not to be dismissed lightly. 
The trouble in the case of rigid polymers (rods) may in fact come from the usual assumption that the mean-field is not a local one, but the related correlation length increases indefinitely, anticipating the existence of a transition to a long-range ordered phase. This assumption is not justified in the case of hard rods on the lattice, where the correlation length does not diverge, but remains finite and in the order of the length of the rod [13] (i.e. the appearance of ordered domains of size $N$ ) thus prohibiting the applicability of common mean-field concepts.

It is important to note that our main conclusion on the non-existence of the nematic static for lattice rods or polymers does not affect the mean-field results concerning the temperature induced configurational transition of polymers between their flexible (coil-like) and their rigid (rod-like) state found in previous works $[3,4,7]$. This problem is characterized by a different correlation length $\xi_{N}$ as compared with the problem of long-range orientational order. There the temperature dependent length scale $\sqrt{N}<\xi_{N}(T)<N$ (which is essentially the average end-to-end distance of the polymer in the coil-like and in the rod-like state respectively) is comparable to the cooperative correlation length $\xi_{r}(T)$ manifested in the appearance of ordered domains of size $\xi_{r}$, as observed in computer experiments [13]. Hence mean-field methods should be applicable to the problem of coil to rod transitions induced by local orientational ordering, and consequently the predicted $[3,4,7]$ configurational transitions in the limit of infinitely long chains seems to be genuine.

The result given above are restriced to two dimensions. But it seems unlikely that the threedimensional case (e.g. hard rods on the simple cubic lattice) would be qualitatively different by exhibiting a nematic phase. This is supported by the fact, that even for the smallest system $L / N=1$, which is an upper bound for the orientational order parameter of larger systems $L / N>1$, the order parameter is $\langle s\rangle \leqslant 1 / 2$ (especially $\langle s\rangle=1 / 4$ for $N \rightarrow \infty$ ), which seems to preclude a nematic state with $\langle s\rangle \simeq 1$ for large systems $L / N>1$. The order parameter is in three dimensions defined as

$$
\langle s\rangle=(3\langle f\rangle-1) / 2
$$

where $\langle f\rangle$ is the fraction of rods parallel to the director defined as the direction of the majority of rods at a given configuration.

$$
\langle f\rangle=3\left[2 \sum_{k=0}^{N / 2}\left(\begin{array}{l}
N \\
k
\end{array}\right)(1-k / N)-\left(\begin{array}{c}
N \\
N / 2
\end{array}\right) / 2-1\right] / Z_{N}
$$

for $N$ even, and $Z_{N}$ is the total number of configurations

$$
Z=3\left(2^{N}-1\right)
$$

Exact enumerations of cubic lattice systems are currently almost impossible, even for $L / N=2$, due to serious storage problems.

Conclusions inferred from present strict lattice results to continuum models for rods are questionable. As a matter of fact, mean-field treatments of quasi-continuum models have revealed a nematic phase in the closed packed density limit if the rod axial ratios are larger than 6.4 and 4.3 in three [14] and two [15] dimensions respectively. The relation between-models with continuous and discrete distribution of orientation are not yet understood.

Finally it should be noted that local orientation dependent forces between adjacent chain segments actually lead to a phase transition between the isotropic and the nematic state for polymers on the square and the cubic lattice [16]. 


\section{References}

[1] Cotter, M. A., Mol. Cryst. Liq. Cryst. 35 (1976) 33.

[2] Alben, R., Mol. Cryst. Liq. Cryst. 13 (1971) 193.

[3] Di Marzio, E. A., J. Chem. Phys. 35 (1961) 658.

[4] Flory, P. J., Proc. R. Soc. A 234 (1956) 60 ; Proc. Natl. Acad. Sci. 79 (1982) 4510.

[5] NaGle, J. F., Proc. R. Soc. A 337 (1974) 569.

[6] Gujrati, P. D., Goldstein, M. J., J. Chem. Phys. 74 (1981) 2596.

[7] Ronca, G., J. Chem. Phys. 79 (1983) 6326.

[8] ONSAGER, L., Ann. N.Y. Acad. Sci. 51 (1949) 627.

[9] Flory, P. J., Proc. R. Soc. A 234 (1956) 73 ; Adv. Polymer. Sci. 59 (1984) 1.

[10] FISHER, M. E., Phys. Rev. 124 (1961) 1664.

[11] Kasteleyn, P. W., Physica 27 (1961) 1208.

[12] LIEB, E. H., J. Math. Phys. 8 (1967) 2339.

[13] Baumgärtner, A., J. Phys. A 17 (1984) L975.

[14] Flory, P. J., Ronca, G., Mol. Cryst. Liq. Cryst. 54 (1979) 289.

[15] RonCA, G., private communication.

[16] Baumgärtner, A., to be published. 